\title{
BACKGROUND AND STIMULUS-INDUCED PATTERNS OF HIGH METABOLIC ACTIVITY IN THE VISUAL CORTEX (AREA 17) OF THE SQUIRREL AND MACAQUE MONKEY ${ }^{1}$
}

\author{
ALLEN L. HUMPHREY ${ }^{2}$ AND ANITA E. HENDRICKSON \\ Department of Ophthalmology, University of Washington School of Medicine, Seattle, Washington 98195
}

Received March 12, 1982; Revised September 9, 1982; Accepted September 10, 1982

\begin{abstract}
We have used 2-deoxy-D- $\left[{ }^{14} \mathrm{C}\right]$ glucose (2-DG) autoradiography and cytochrome oxidase histochemistry to examine background and stimulus-induced patterns of metabolic activity in monkey striate cortex. In squirrel monkeys (Saimiri sciureus) that binocularly or monocularly viewed diffuse white light or binocularly viewed bars of many orientations and spatial frequencies, 2-DG consumption was not uniform across the cortex bul consisted of regularly spaced radial zones of high uptake. The zones extended through all laminae except IVc $\beta$ and, when viewed tangentially, formed separate patches $500 \mu \mathrm{m}$ apart. The cytochrome oxidase stain in these animals also revealed patches of high metabolism which coincided with the 2-DG patches. Squirrel monkeys binocularly viewing vertical stripes showed parallel bands of increased 2-DG uptake in the cortex, while the cytochrome label in these animals remained patchy. When monkeys were kept in the dark during 2-DG exposure, 2-DGlabeled patches were not seen but cytochrome oxidase-positive patches remained.

In macaque (Macaca nemestrina) monkeys, binocular stimulation with many orientations and spatial frequencies produced radial zones of high 2-DG uptake in layers I to IVa and VI. When viewed tangentially, these zones formed a dots-in-rows pattern with a spacing of $350 \times 500 \mu \mathrm{m}$; cytochrome oxidase staining produced an identical pattern. Macaca differed from Saimiri in that monocular stimulation labeled alternate rows. These results indicate that there are radial zones of high background metabolism across squirrel and macaque monkey striate cortex. In Saimiri these zones do not appear to be related to an eye dominance system, while in Macaca they do. The presence of these zones of high metabolism may complicate the interpretation of 2-DG autoradiographs that result from specific visual stimuli.
\end{abstract}

Deoxyglucose (2-DG) autoradiography is a potentially powerful neuroanatomical technique, for it allows direct anatomical visualization of stimulus-induced alterations in neuronal activity (Sokoloff et al., 1977). Its main potential for studying visual cortex is that different stimulus conditions may generate unique patterns of 2-DG uptake which can suggest how cells that process different

'This work was funded by National Institutes of Health Grants EY07013, EY-01208, EY-01730 and EY-03321, and in part by Grants RR00166 and HD02274. We wish to thank Dr. Jennifer Lund for her useful input during the early stages of this study. We also thank Dr. John Dineen, Jim Foltz, Brad Clifton, and DeVeta Ludelle Moe for technical assistance, and Julie Seng for manuscript preparation. Finally, we thank Dr. Terry Allen for her assistance on the computerized image processing system at the Department of Electrical and Computer Engineering, Drexel University in Philadelphia.

${ }^{2}$ To whom correspondence should be addressed at his present address: Department of Neurobiology and Behavior, Graeluate Biology Building, State University of New York, Stony Brook, NY 11794. visual modalities such as ocularity, orientation, or color are distributed within the cortex. The technique also labels the distribution of a particular modality over much broader regions of cortex than is obtainable with microelectrode recordings (e.g., see Hubel et al., 1978; Humphrey and Norton, 1980; Humphrey et al., 1980).

Despite these advantages, the results from 2-DG experiments in monkey visual cortex have not always been easy to reconcile with those from other techniques. Trans-synaptic autoradiography (Hubel et al., 1977; Hendrickson et al., 1978), Nauta (Hubel and Wiesel, 1972) and Liesegang silver stains (LeVay et al., 1975), and neurophysiology (Hubel and Wiesel, 1968) have all shown the presence of a repetitive alteration in eye input to layer IV of Macaca monkeys. These have been called ocular dominance columns. When one eye is stimulated, 2-DG labeling also showed ocular dominance columns which extend to all layers above and below layer IVc in the same manner as suggested by electrophysiological recording (Kennedy et al., 1976; Hubel et al., 1978; Hen- 
drickson and Wilson, 1979). When the same techniques were applied to New World monkeys such as the squirrel monkey (Saimiri sciureus), quite different results were obtained. Trans-synaptic autoradiography showed that the lateral geniculate nucleus (LGN) afferents which represent each eye terminate in layer IV with considerable overlap (Hubel et al., 1976; Tigges et al., 1977; Hendrickson et al., 1978) and electrophysiological recordings showed that most cells in layer IV of Saimiri are binocular (Hubel and Wiesel, 1978). These results have led several workers to conclude that squirrel monkeys lack ocular dominance columns (for review, see Hendrickson et al., 1978). However, when deoxyglucose labeling was done in squirrel monkeys stimulated monocularly with all stimulus orientations, radial zones of increased 2-DG uptake were found above and below a uniformly labeled layer IV (Hendrickson and Wilson, 1979). This led us to question whether these radial zones outside of layer IV represented a type of hidden ocular dominance column system in Saimiri or some other cortical system unrelated to ocular dominance. As well, Hendrickson and Wilson (1979, unpublished observations) found in macaque monkeys binocularly stimulated with all orientations that regularly spaced radial zones of high deoxyglucose uptake were still present across striate cortex. Because these animals viewed many orientations through both eyes, it was not clear just what these radial zones represented in Macaca, especially in view of a previous report which suggested that similar binocular stimulus conditions resulted in uniform labeling above layer IV (Kennedy et al., 1976).

The above uncertainties led us to systemically re-examine background and stimulus-induced patterns of 2DG uptake in area 17 (striate cortex) of squirrel and macaque monkeys. Because Wong-Riley $(1976,1979)$ has provided strong evidence that high cytochrome oxidase enzyme levels in the brain indicate regions of high oxidative metabolism, we also stained the same cortical tissue for cytochrome oxidase to compare the patterns of the two labels. Using these two different labels, our results in both monkeys show that area 17 contains regularly repeating radial zones of high metabolism and that the presence of this base line activity can complicate the interpretation of 2-DG autoradiographs that are produced by specific visual stimulation. The results from the experiments in the squirrel monkey suggest that these zones are not associated with an ocular dominance column system. In Macaca, on the other hand, they may be an extension of the eye dominance system outside of layer IV. A preliminary report of this work has appeared previously (Humphrey and Hendrickson, 1980).

\section{Materials and Methods}

Ten adult squirrel monkeys (Saimiri sciureus), 0.5 to $1.0 \mathrm{~kg}$, and two juvenile macaque monkeys (Macaca nemestrina), 2.0 to $2.5 \mathrm{~kg}$, were used. On the day of the experiment, the animal was tranquilized with ketamine hydrochloride $(6$ to $30 \mathrm{mg} / \mathrm{kg}$ ) and then was anesthetized with 1 to $3 \%$ halothane while the femoral vein was quickly cannulated. The animal was then placed in a modified chair, so constructed that the torso was held snugly in an upright position and the limbs were gently held out of the field of view. A bolt held in a rigid frame was then screwed into a hex-nut which had been permanently attached to the top of the skull 3 to 7 days earlier; this bolt-nut arrangement immobilized the head. If required for the stimulus conditions, one eyelid was then sutured closed and/or covered with a black patch. Within 3 to 4 $\mathrm{hr}$, the animal became completely alert and the 2-DG experiment commenced. Indices of alertness were a prompt visual orienting and following behavior and a strong, maintained grasp reflex. This awake, unparalyzed preparation was used so that normal, binocular vergence would be achieved voluntarily by the monkey, and the animal would be in a normal state of alertness.

Animals were given intravenous pulse injections of 2deoxy-D- $\left[{ }^{14} \mathrm{C}\right]$ glucose in tracer amounts $(90$ to $150 \mu \mathrm{Ci} / \mathrm{kg}$ in 0.25 to $1.0 \mathrm{ml}$ of sterile saline; specific activity, 50 to 56 $\mathrm{mCi} / \mathrm{mm}$; Amersham) while being visually stimulated for $45 \mathrm{~min}$. Stimuli included a complex laboratory scene, a patterned stimulus, diffuse light, or the absence of light (bilateral lid suture with black blindfold). For Saimiri, the patterned stimulus consisted of a large photopically illuminated screen containing parallel, black and white, rectangular stripes of many spatial frequencies which was shifted continuously from side to side perpendicular to the axis of stripe orientation. Stripe orientation remained constant for some animals or was changed every $30 \mathrm{sec}$ for others (all orientation condition). The screen was $1.5 \mathrm{~m}$ on a side and covered the animal's visual field from the center of gaze to an azimuth of $60^{\circ}$ and an elevation of $\pm 45^{\circ}$. When held at $42 \mathrm{~cm}$ from the animal, the stripes subtended $4^{\prime}$ to a $2.5^{\circ}$ visual angle (average angle $=25^{\prime}$ arc; spatial frequencies $=0.2$ to 7.5 cycles/ degree). To ensure that the animal viewed the stimulus screen primarily, curtains were hung from ceiling to floor to mask extraneous stimuli. Because the animal had normal eye movements during the visual stimulation, the experimenter constantly monitored the animal's direction of gaze through a pinhole in the screen and, when necessary, shifted the screen position to maintain screen center with center of gaze. For the animals stimulated with diffuse light, either a large, photopically illuminated (tungsten source) white screen was used in a similar manner, or a photopically illuminated white Plexiglas hemisphere $(76 \mathrm{~cm}$ in diameter) which covered the animal's whole visual field was used. To examine 2-DG uptake in the absence of light, in one adult squirrel monkey both eyelids were sutured and its eyes were covered with a black tape mask during the stimulation period.

'The two Macaca were allowed to view a complex laboratory scene which contained moving people. The animal also was moved about the room so that it viewed different aspects of the scene during the stimulus period.

Following the stimulation the animals were deeply anesthetized with an overdose of intravenous Pentothal and immediately were perfused with $500 \mathrm{ml}$ of $4 \%$ paraformaldehyde in $0.1 \mathrm{~m}$ phosphate buffer. The visual cortex was then removed, blocked, and frozen at $-75^{\circ} \mathrm{C}$ in Freon chilled in liquid nitrogen. Serial sections $(30 \mu \mathrm{m})$ were cut at or below $-20^{\circ} \mathrm{C}$ in a cryostat, picked up on coverslips, and quickly dried on a $60^{\circ} \mathrm{C}$ hot plate. Selected sections were freeze-dried onto coverslips by leaving them in the cryostat for 30 to $48 \mathrm{hr}$. All autoradi- 
ographs were made by exposing the sections to DuPont Cronex Mammography film for 2 to 5 weeks at room temperature.

For the demonstration of cytochrome oxidase, freshly cut frozen or freeze-dried sections were reacted free floating in $50 \mathrm{mg}$ of diaminobenzidine (Sigma Chemical Co.), $15 \mathrm{mg}$ of cytochrome $c$ (Sigma, type III), and $4 \mathrm{gm}$ of sucrose for each $100 \mathrm{ml}$ of $0.1 \mathrm{M}$ phosphate buffer $(\mathrm{pH}$ 7.4). Sections were reacted for 0.5 to $24 \mathrm{hr}$ at $37^{\circ} \mathrm{C}$ with frequent changes of the medium. They were rinsed in buffer, mounted on slides, dried, dehydrated, and coverslipped. This method is modified from that of WongRiley (1979).

While the aim of this study was to examine qualitatively the patterns of 2-DG uptake resulting from different stimulus conditions, selected sections of squirrel monkey brains were also examined quantitatively to estimate the degree of fluctuation in 2-DG uptake within each condition. Tangential sections from the operculun of area 17 , cut at the level of midlayer III, were exposed to $\mathrm{x}$-ray film for a constant exposure time. Then the absolute optical densities of adjacent regions of high and low 2DG uptake were measured in each section using a computerized image processing system. The measurement window was a square $185 \mu \mathrm{m}$ on a side. For each animal, an approximation to the degree of fluctuation in 2-DG uptake was expressed as the ratio of the mean optical density of regions of high uptake to the mean density of regions of lower uptake. This method of approximation does not readily allow one to compare fluctuation levels between animals because it does not take into account, among other things, interanimal differences in overall levels of 2-DG utilization. Such interanimal comparisons must await more refined studies.

\section{Results}

\section{Saimiri: Deoxyglucose experiments}

Stimulation with one orientation viewed binocularly. Previous studies in the tree shrew, cat, and macaque monkey have shown that binocular visual stimulation with vertically or horizontally oriented stripes yields a band- or lattice-like pattern of 2-DG uptake across striate

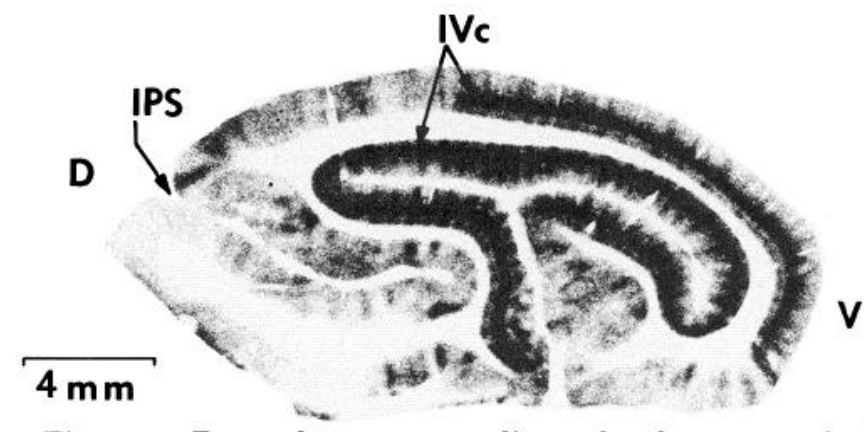

Figure 1. Deoxyglucose autoradiograph of a parasagittal section through the right visual cortex from a squirrel monkey that binocularly viewed vertical stripes. Lamina IVc in area 17 is evident as a continuous band of very high 2 -DG uptake, but radial zones of heightened uptake outside IVc are present throughout area 17. IPS, interparietal sulcus; $D$, dorsal; $V$, ventral. In this and the following figures, the scale is uncorrected for tissue shrinkage or expansion.
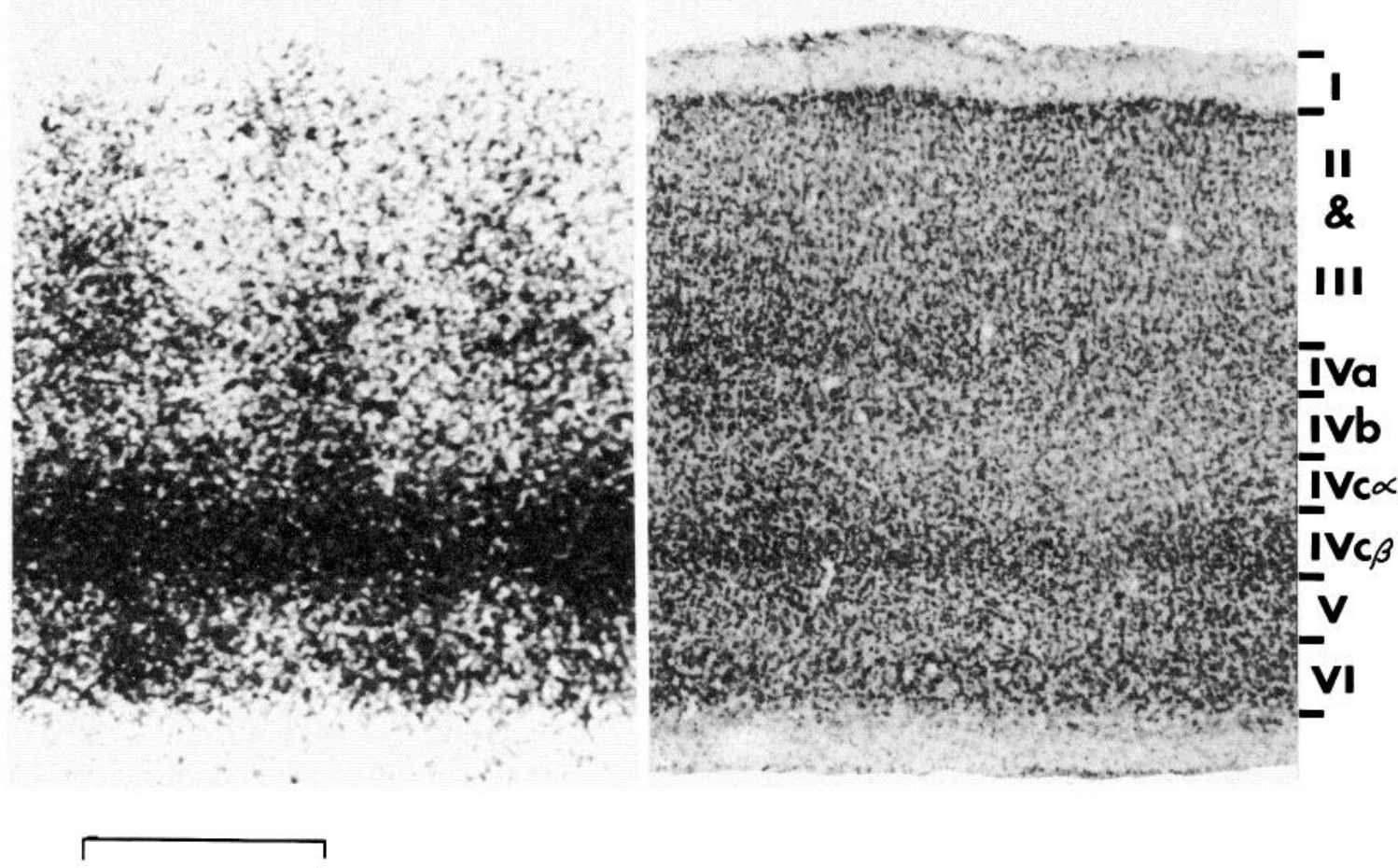

Figure 2. Comparison of the laminar distribution of deoxyglucose label in squirrel monkey striate cortex with the distribution of Nissl-stained cell bodies. On the left is an autoradiograph of a coronal section from a monkey stimulated binocularly with vertical stripes. The autoradiograph is from the same monkey as that illustrated in Figure 1 and is taken from the roof of the calcarine cortex a number of sections away from that shown in Figure 1. The same section stained for cell bodies is on the right. The laminar numbering scheme of Lund (1973) has been used. Bar, $500 \mu \mathrm{m}$. 
cortex (Stryker et al., 1977; Hubel et al., 1978; Albus, 1979; Flood and Coleman, 1979; Humphrey et al., 1980; Horton and Hubel, 1981; Tootell and Silverman, 1981). Three squirrel monkeys received 2-DG while binocularly viewing vertically oriented stripes on the stimulus screen. Figure 1 shows the resulting laminar uptake in an autoradiograph of a parasagittal section through the right visual cortex of one of these animals. The laminar variations in 2-DG uptake seen in this section were similar in all of the animals that received light stimulation and were found throughout all regions of striate cortex. Area 17 is delimited by layer IVc, which is continuously and densely labeled throughout all of this animal's left and right striate cortex. Although not obvious in most crosssections through area 17 , a relatively continuous band of increased 2-DG uptake is also present within layer IVa. This continuity is most evident in autoradiographs of tangential sections (see below). The precise correspondence between the Nissl-stained cortical laminae and the laminar variations in 2-DG uptake are illustrated in Figure 2. In all of the cases, deoxyglucose uptake within the layer IV complex was highest in IVc, lowest in IVb, and generally intermediate in IVa.

Superimposed across this laminar pattern of 2-DG uptake are radial zones of increased 2-DG uptake which extend from the cortical surface to white matter and which are most distinct in layers II to IVa and VI (Figs. 1 and 2). The radial zones are more heavily labeled in the mushroom-shaped calcarine cortex than on the exposed opercular regions of area 17 . We do not interpret this difference, however, as a sign that the opercular cortex was poorly stimulated since this region represents the central $4^{\circ}$ of visual field (Cowey, 1964), and therefore it must have been well stimulated by the striped screen.

Despite the lower density of the radial zones along the operculum, their pattern of distribution in tangential section is quite clear. When viewed from above, these radial zones of high uptake form a bandlike or zig-zag pattern as shown in Figure 3. The bands are most distinct in layers II and III (Fig. $3 A$ ); they are wavy but locally parallel with an average center-to-center spacing of 500 $\mu \mathrm{m}(\mathrm{SD}=90 \mu \mathrm{m})$. Adjacent bands often merge with each other or terminate abruptly. Interestingly, the level of 2DG uptake along the bands is not uniform; instead, small circular regions of very high 2-DG uptake ("hot spots") stand out along the well labeled bands. The average optical density of the bands of heightened uptake in this animal is $0.72(\mathrm{SD}=0.16)$, while that for the interband regions is $0.45(\mathrm{SD}=0.09)$, yielding a ratio of 1.60 .

These tangential sections also show that the band pattern persists within layers IVa and IVb (Fig. $3 B$ ) and even faintly within the upper part of IVc $\alpha$ (Fig. $3 C$ ). Although not shown, the 2-DG label within layer IVc $\beta$ appears completely uniform. Within layers V and VI the bands can once again be seen, although they appear more patchy than the bands above layer IV. Thus, a bandlike distribution of high 2-DG uptake is present across area 17 of Saimiri after binocular stimulation with oriented stripes.

Stimulation with diffuse light viewed monocularly. Hendrickson and Wilson (1979) demonstrated that when a squirrel monkey monocularly viewed many orientations, the radial and laminar distribution of 2-DG uptake was identical to that seen here in Figure 1. However, in tangential sections the zones appeared as separate patches $500 \mu \mathrm{m}$ apart, rather than as bands. Because the stimuli used in these earlier experiments consisted of a complex, active laboratory scene, the possibility existed that some stimulus orientations were more represented in the scene than others or that the complexity of the visual scene introduced some other unknown stimulus parameters. To check for these possibilities, we examined the pattern of 2-DG uptake in one squirrel monkey that
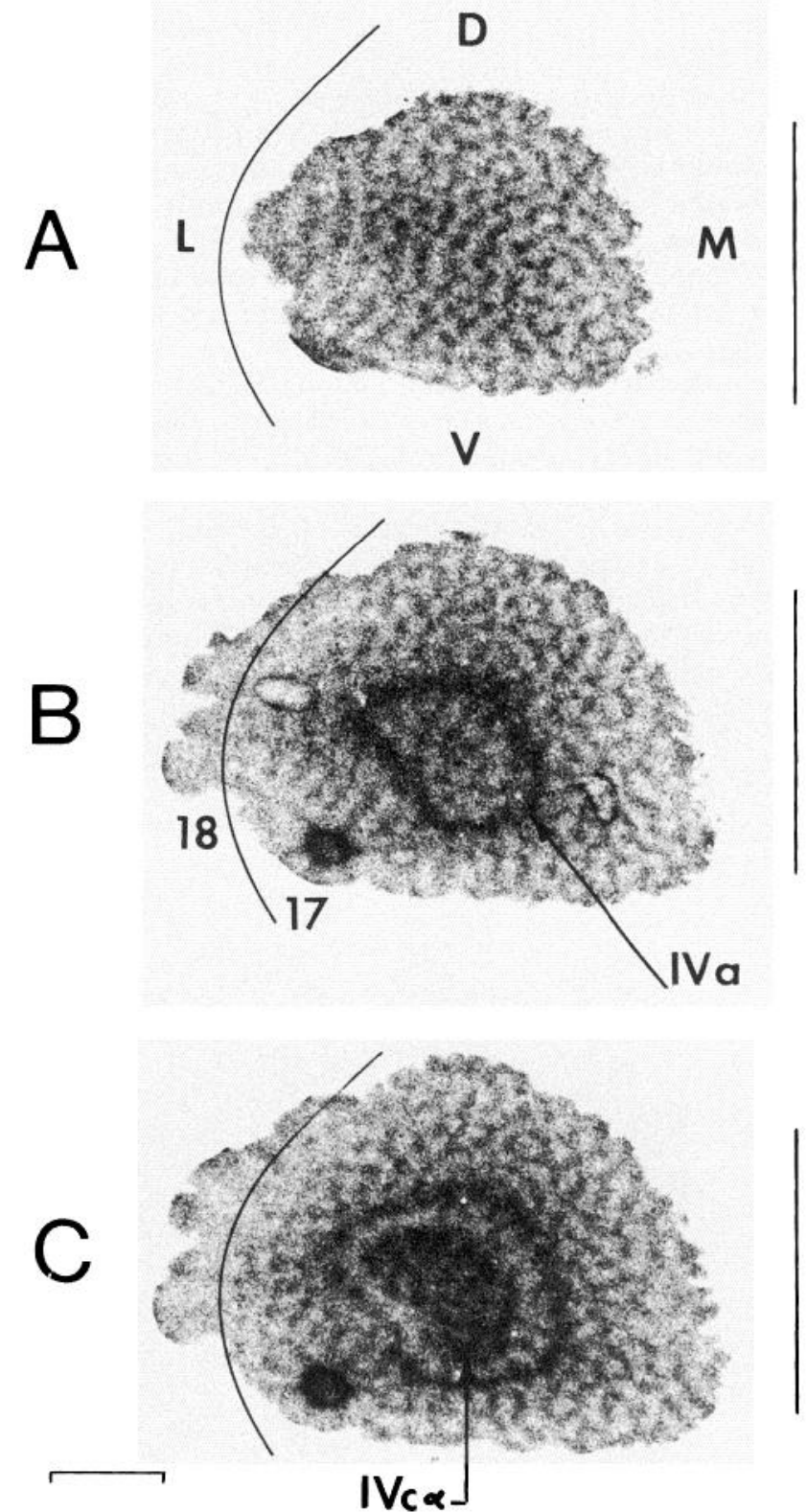

Figure 3. Deoxyglucose autoradiographs of three progressively deeper tangential sections through the opercular region of the left striate cortex from a squirrel monkey stimulated with vertical stripes. In $A$, the plane of tangential section is primarily through laminae II and III; in $B$, it is primarily through laminae III to IVb; and in $C$, it is primarily through laminae III to IVc. Note the complex, bandlike pattern of high 2-DG uptake. The curved lines on the left of each autoradiograph indicate the approximate position of the area 17-18 border; the vertical lines on the right indicate the midline. $L$, lateral; $D$, dorsal; $M$, medial; $V$, ventral. Scale bar, $2 \mathrm{~mm}$. 


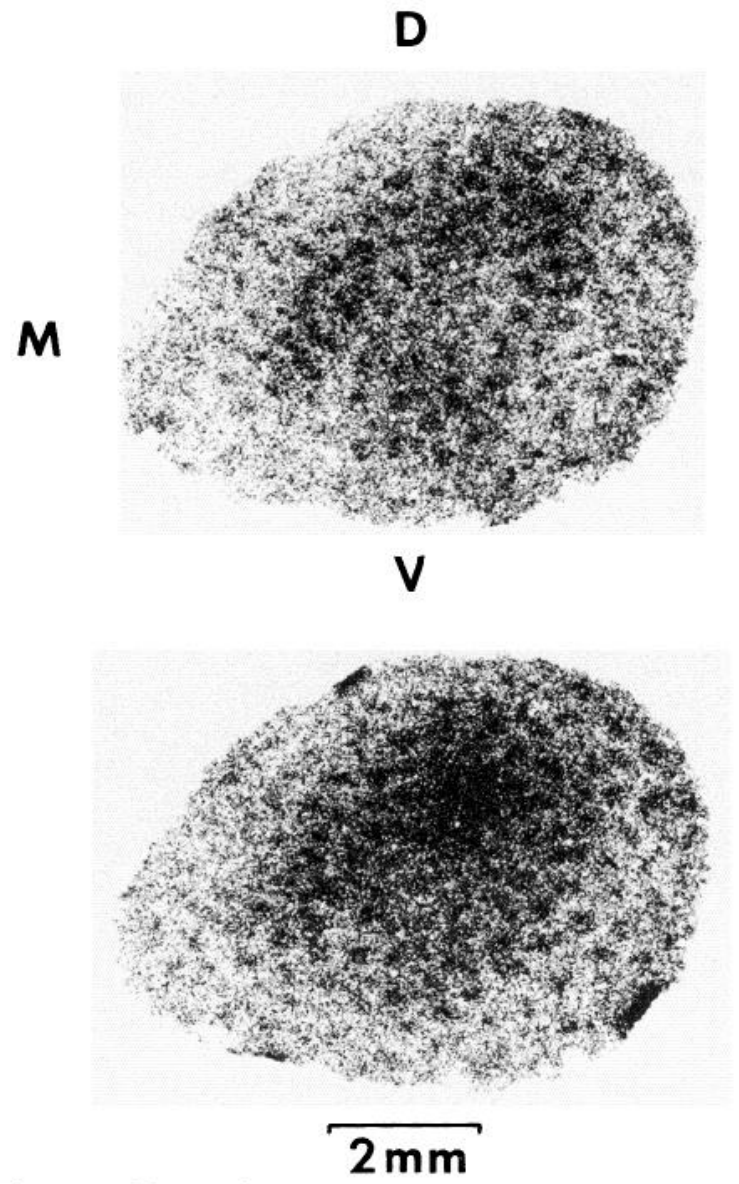

Figure 4. Deoxyglucose autoradiographs of two successive tangential sections, primarily through lamina III of the right opercular cortex of a squirrel monkey monocularly stimulated by diffuse light. Note the patches of high 2-DG uptake. $M$, medial; $D$, dorsal; $L$, lateral; $V$, ventral.

was monocularly stimulated with diffuse light in a Plexiglas hemisphere. Although this eliminated orientation, color, and pattern from the stimulus condition, our results are identical to those of Hendrickson and Wilson (1979). Figure 4 shows autoradiographs of two successive tangential sections through layers II and III of the striate cortex ipsilateral to the open eye. Distinct patches of high 2-DG uptake are spaced about $500 \mu \mathrm{m}$ apart (SD $=105 \mu \mathrm{m})$. The patches appear in layer II to the upper part of IVa and in layers V and VI. The same pattern is present in both hemispheres. Across layer III, the mean optical density of the patches is $0.85(\mathrm{SD}=0.09)$, while that for the interpatch regions of lower density is 0.66 $(\mathrm{SD}=0.12$ ), yielding a ratio of 1.29 . The obvious interpretation of these patchy zones is that they represent ocular dominance columns, while the wavy bands seen in the animal illustrated in Figure 3 reflect the orientation system. If ocularity were eliminated by having both eyes stimulated and orientation were eliminated by having all orientations present in the stimulus light, a uniform pattern of 2-DG uptake would be expected. The next experiment was designed to test the prediction.

Stimulation with all orientations viewed binocularly. Three squirrel monkeys were stimulated binocularly with the striped screen while the screen orientation was changed every $30 \mathrm{sec}$ to equally present orientations of horizontal, vertical, and oblique. Figure 5 shows the resulting autoradiographs from two successive tangential sections through layers II and III of the left striate cortex of one of these animals. Regularly spaced patches of high 2-DG uptake which have a spacing of $500 \mu \mathrm{m}$ are still present and they appear in the same laminae as the monocularly stimulated animal above. The mean optical density of the patches across layer III is $0.78(\mathrm{SD}=0.08)$, while that for the interpatch regions is $0.50(\mathrm{SD}=0.15)$, yielding a ratio of 1.56 . These patches were unexpected because similar experimental conditions in Macaca were reported to have produced a uniform distribution of uptake across striate cortex (Kennedy et al., 1976). The continued presence of these patches in squirrel monkey suggested that there might be something unique about the stimulus stripes, such as their spatial frequency, to which particular groups of cortical neurons responded.

Stimulation with diffuse light viewed binocularly. In an attempt to eliminate as much spatial frequency information as possible from the visual scene, two animals were stimulated binocularly with an unpatterned diffuse light while they viewed either a uniformly white screen or the Plexiglas hemisphere. We assumed that this eliminated virtually all but the lowest spatial frequencies. The results from both viewing conditions were the same. Autoradiographs of two tangential sections from the
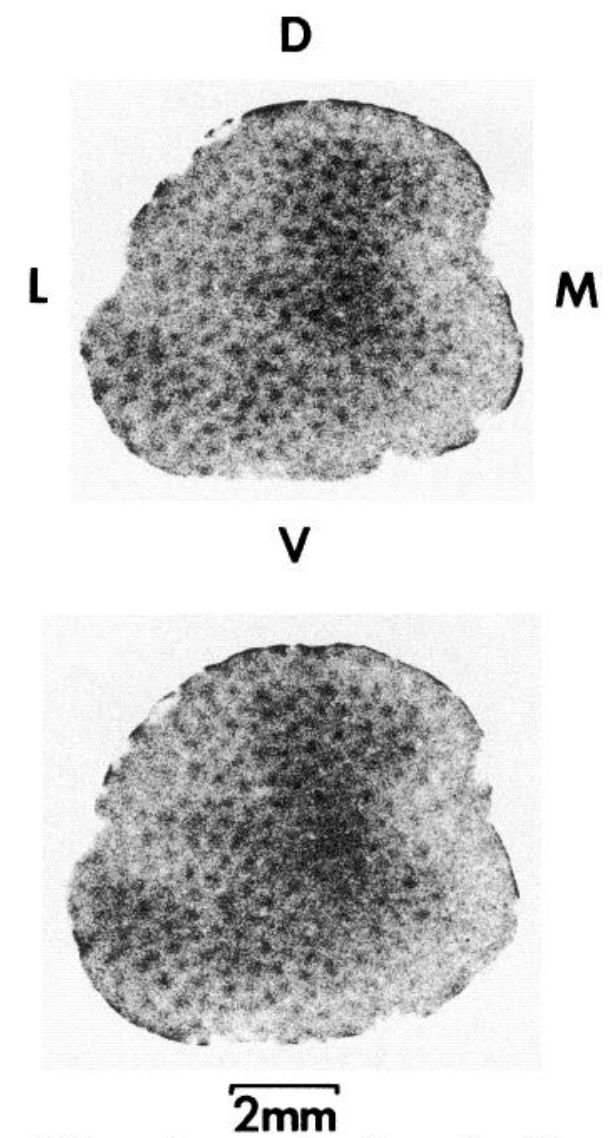

Figure 5. Deoxyglucose autoradiographs of two successive tangential sections primarily through deep lamina III of the left opercular cortex of a squirrel monkey stimulated binocularly with all orientations. Distinct patches of high 2-DG uptake are clearly visible. $L$, lateral; $D$; dorsal; $M$, medial; $V$, ventral. 

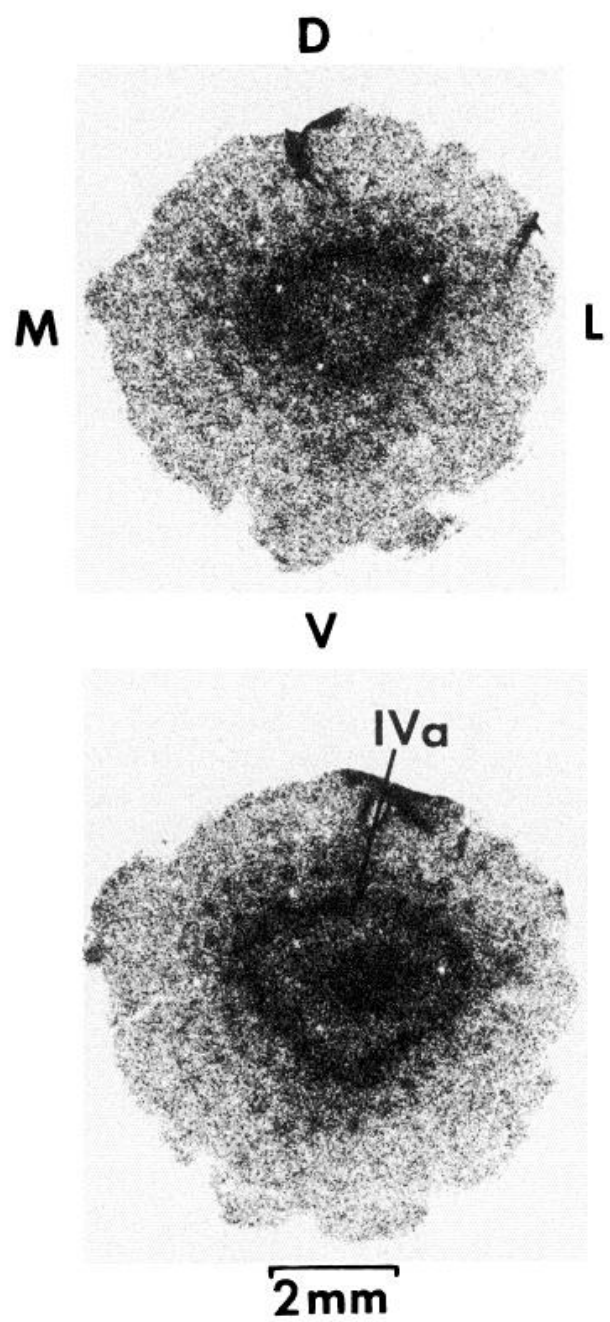

animal that viewed the screen are shown in Figure 6 . Labeled patches having a spacing of about $500 \mu \mathrm{m}$ (SD $=85 \mu \mathrm{m})$ are still present in and above layer IVa while layer IVc appears continuously labeled. In both animals, visual inspection of the autoradiographs suggested that the difference in uptake between the patch and interpatch regions might be lower than in the previous animals since the patches in layer III (Fig. 6) do not appear to stand out as clearly as in the previous animals. However, measurements of the optical densities in the autoradiographs illustrated in Figure 6 revealed a patch/interpatch ratio of 1.50 , with the density of the patches being $0.69(\mathrm{SD}=0.07)$ and the interpatches being $0.46(\mathrm{SD}=$ 0.11 ). This ratio may be spuriously high because it was more difficult to visualize many of the patches for measurement in this animal and only the patches of higher contrast were measured. Despite the uncertainty in the degree of fluctuation in this animal, it is clear that diffuse light lacking pattern or orientation when viewed through one or both eyes is sufficient to generate patches of heightened metabolic activity across squirrel monkey striate cortex.

Deoxyglucose uptake in the absence of light. The final experiment was designed to examine what patterns of 2DG uptake exist in the absence of light; i.e., dark uptake. An adult squirrel monkey was binocularly lid sutured 3

Figure 6. Deoxyglucose autoradiographs of two tangential sections from a squirrel monkey binocularly stimulated with diffuse light. The upper section encompasses laminae I through IVb of the opercular cortex, while the lower one, a few sections deeper, encompasses laminae I through IVc. Patches of increased 2-DG uptake are most evident in the lower half of lamina III. $M$, medial; $D$, dorsal; $L$, lateral; $V$, ventral.
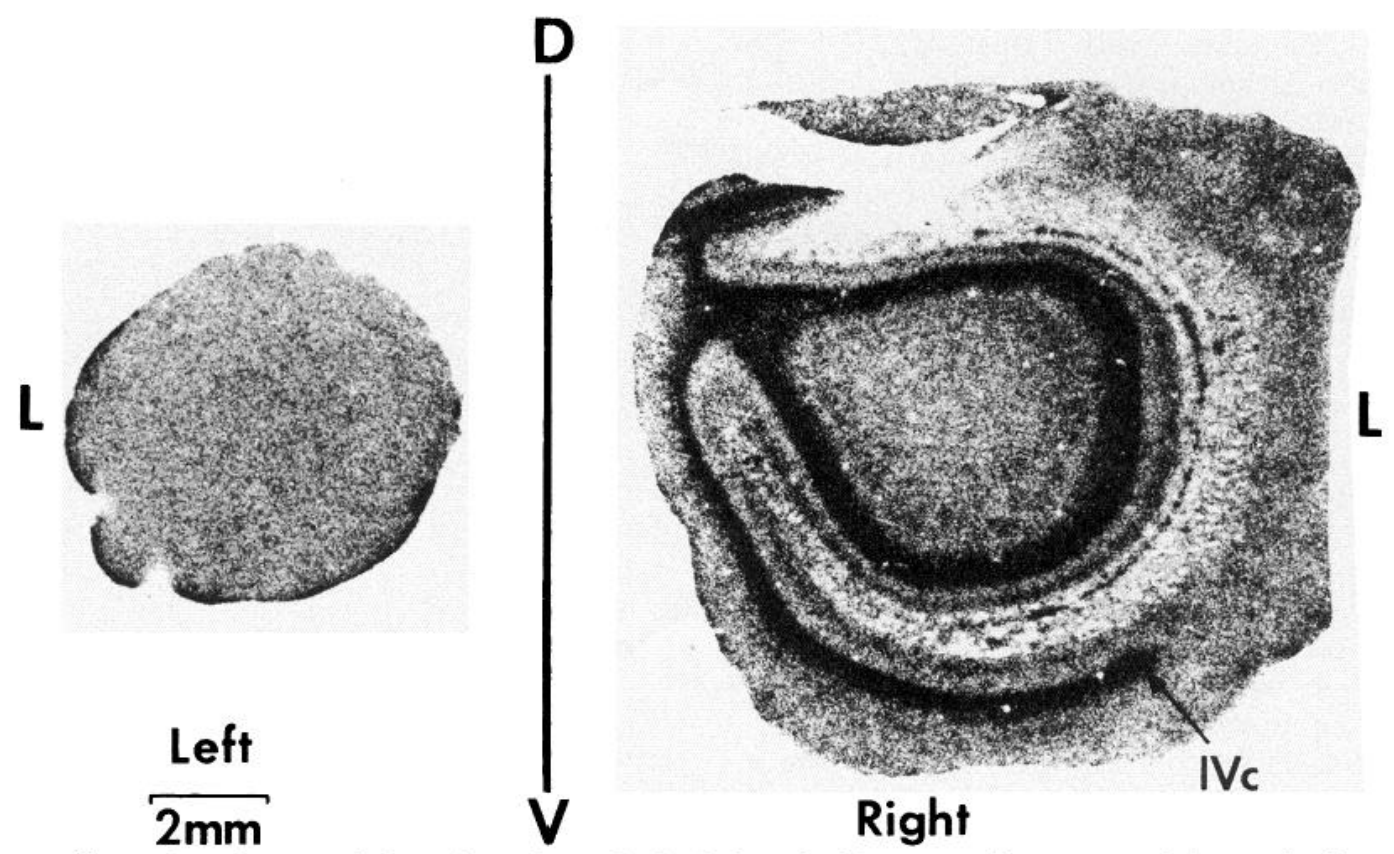

Figure 7. Autoradiographs of tangential sections through the left and right opercular cortex of the squirrel monkey that was binocularly lid sutured before being injected with deoxyglucose. The left section is primarily through lamina III; the right section cuts through the caudal opercular cortex and the underlying calcarine cortex. Note the absence of radial zones or patches of heightened 2-DG uptake in these sections, but the heavy labeling of layer IVc persists. $L$, lateral; $D$, dorsal; $V$, ventral. 


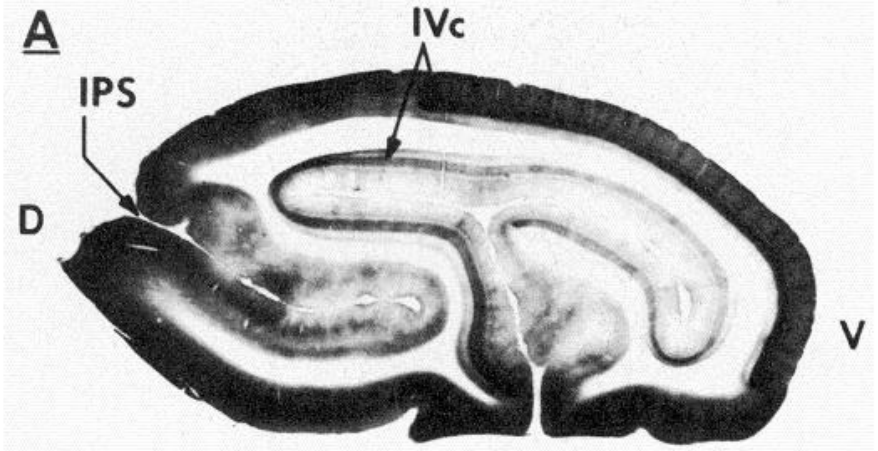

$\underline{\mathbf{B}}$

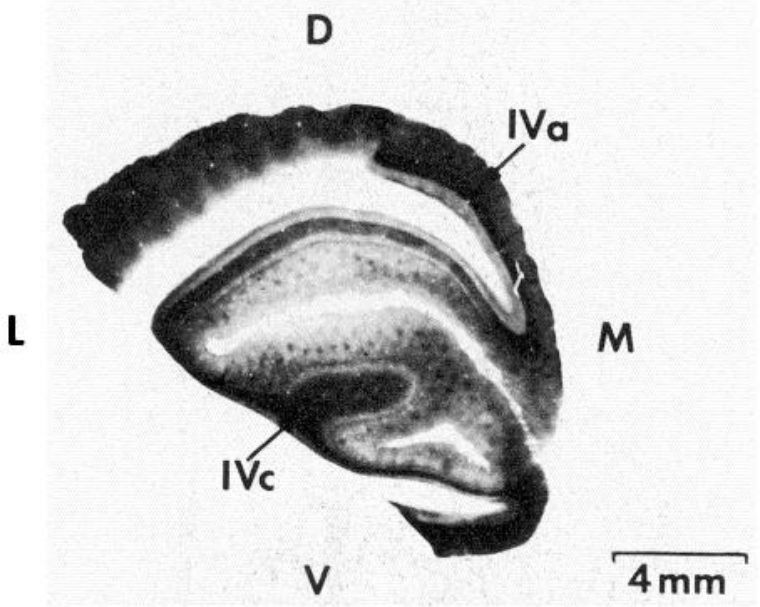

Figure 8. Patterns of cytochrome oxidase reactivity in the striate cortex from the squirrel monkey that was binocularly stimulated with vertical stripes. These sections are from the same squirrel monkey as in Figure 1. A, Parasagittal section through the right visual cortex, near the section shown in Figure 1 . The density of cytochrome oxidase reactivity in this section is much higher on the opercular surface than in calcarine cortex, but this was not typical for other sections and other animals where both regions of area 17 are equally reactive. $B$, Oblique section primarily through the left calcarine cortex illustrating the patches of high cytochrome oxidase activity in lamina III. $D$, dorsal; $V$, ventral; $L$, lateral; $M$, medial,

days before the experiment, and on the day of the experiment the closed lids and surrounding tissue were covered with black tape before the animal was placed in the apparatus. Autoradiographs from the left and right striate cortex are shown in Figure 7. Unlike the previous animals, no patches of high 2-DG uptake are detectable, and the uptake across each layer appears uniform. The layers themselves are well differentiated, with layer IVc being the most heavily labeled.

In summary, our deoxyglucose results in squirrel monkey striate cortex show that there are distinct radial zones of high metabolic activity which are most prominent in layers II to IVa and VI and which occur every $500 \mu \mathrm{m}$. Activity within these zones is dependent upon light stimulation, but the light does not have to contain pattern or orientation information for them to be labeled with 2-DG. Either monocular or binocular stimulation produces the same pattern. Thus, the zones may be thought of as being a basic organizational feature of cortex because they appear to become highly active metabolically under many conditions of light and pattern stimulation. When viewed from above, this pattern usually consists of distinct patches of high deoxyglucose uptake, except following stimulation with stripes of a single orientation when a bandlike pattern of uptake is observed.

\section{Saimiri: Cytochrome oxidase histochemistry}

Sections from each of the above deoxyglucose animals were also reacted for cytochrome oxidase to compare the density distribution of this mitochondrial enzyme with the 2-DG patterns. For all stimulus conditions, including the dark uptake condition, the laminar, radial, and tangential patterns of cytochrome oxidase label were similar throughout all regions of striate cortex. Figure 8 shows two reacted sections from a monkey that was binocularly stimulated with vertical stripes. In this stain, regions of

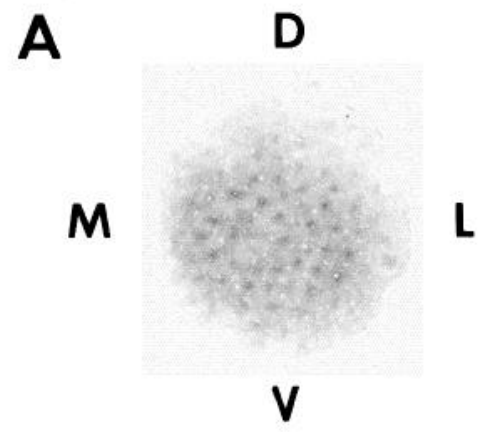

\section{$\overline{3 m m}$}

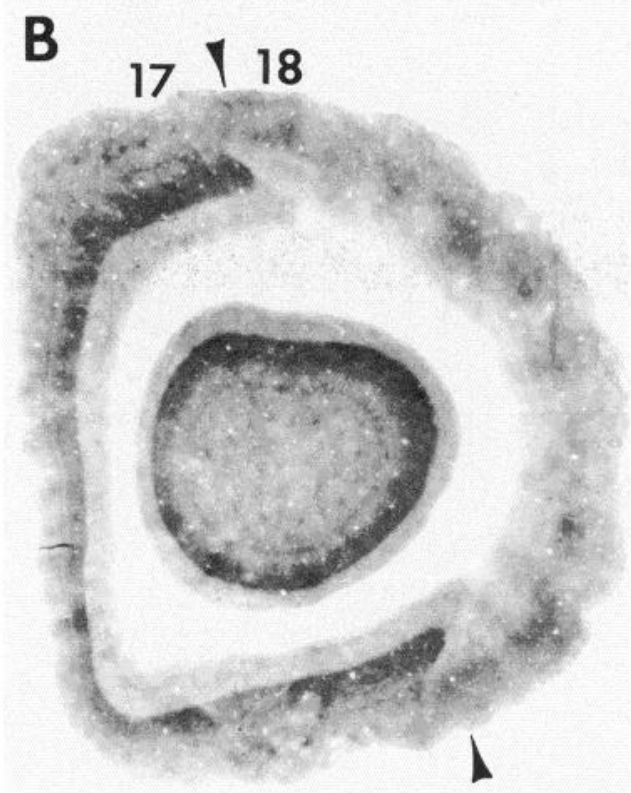

Figure 9. Cytochrome oxidase patterns in tangential sections of the right striate cortex from the animal stimulated in the ganzfeld (Fig. 4). The section in $A$ is mainly through lamina III on the opercular surface; $B$ is a section deeper in the same hemisphere. $M$, medial; $D$, dorsal; $L$, lateral; $V$, ventral. 
$\underline{A}$
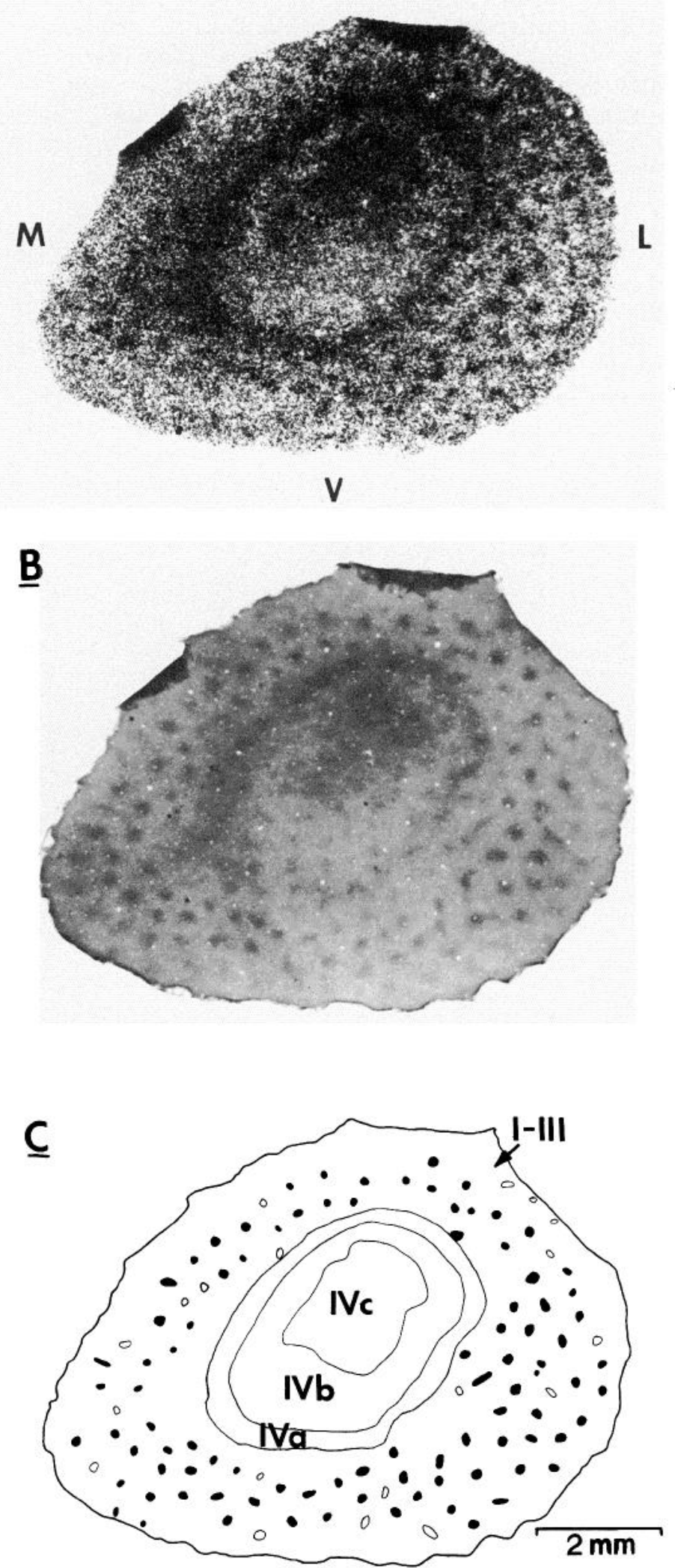

Figure 10. A double labeled tangential section through the right striate cortex of the squirrel monkey that was monocularly stimulated with diffuse light. $A$ shows the 2-DG autoradiograph and $B$ shows the cytochrome oxidase-stained section. $C$ is a drawing indicating the overlap between the two labels. The
M

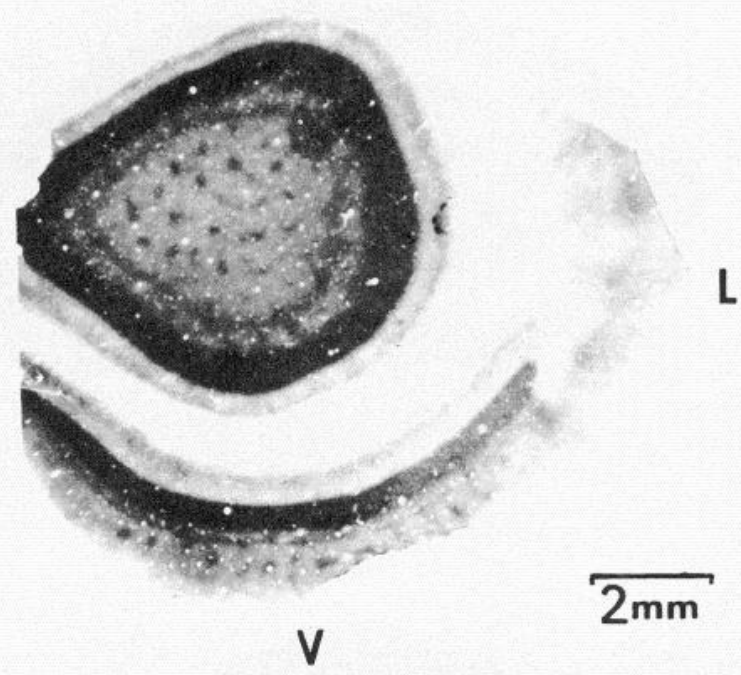

Figure 11. The pattern of cytochrome oxidase label in a tangential section through the right opercular and calcarine cortex of the squirrel monkey that was binocularly lid sutured (Fig. 7). Note the continued presence of patches of high cytochrome oxidase activity. $D$, dorsal; $L$, lateral, $V$, ventral; $M$, medial.

high cytochrome oxidase activity are darker than regions of lower activity. The main constituent of the label appears to be diffusely labeled neuropil. Some stained somata are visible in the sections including large pyramidally shaped cell bodies at the top of layer VI, large cell bodies resembling stellate cells in layers IVb and IVc $\alpha$, and scattered small cell bodies in all layers (not illustrated). Layers IVa and IVc (Fig. 8) are densely and continuously labeled up to the area $17-18$ border, although at higher magnification, layer IVa has a fenestrated appearance (Figs. 10 and 11). The cytochrome oxidase-labeled boundaries match laminar boundaries seen in counterstained Nissl-stained sections.

As in the 2-DG autoradiographs, radial zones of high cytochrome oxidase reactivity are present which are most distinct within layers II and III. While layer IVc is intensely and continuously stained, regions of that layer that are in register with the radial zones above appear somewhat more densely reactive, indicating that the radial zones of high cytochrome oxidase activity extend through layer IVc. The zones also appear to be present in layers V and VI, but they are much less distinct there, compared to the 2-DG label. In tangential section (Fig. $8 B$ ), these zones form separate patches roughly $500 \mu \mathrm{m}$ apart. This patchy cytochrome distribution differs from the bandlike 2-DG pattern in this animal (Fig. 3), although both have the same $500-\mu \mathrm{m}$ spacing. Prestriate cortex also contains radial zones of high cytochrome oxidase activity (Figs. $8 B$ and $9 B$ ). These zones extend through all of the layers and are spaced 800 to $1000 \mu \mathrm{m}$ apart. They are found throughout the visual cortex be-

solid circles in $C$ were labeled by both techniques and constitute $83 \%$ of the identifiable patches. Many of the open circles in $C$ could not be resolved in the autoradiograph. See the text for further discussion. $M$, medial; $D$, dorsal; $L$, lateral; $V$, ventral. 


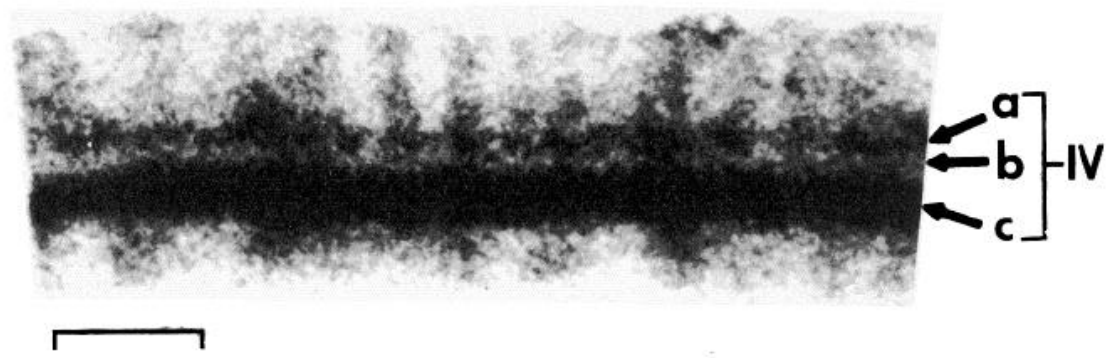

Figure 12. Autoradiograph of a section through the calcarine region of the striate cortex from a macaque monkey that was binocularly stimulated with many orientations. Both the laminar and radial pattern of 2-DG uptake is illustrated. Note the overall similarity in uptake pattern to that seen in the squirrel monkey (Fig. 2), although in this figure layer IVa is more heavily labeled. Scale bar, $1 \mathrm{~mm}$.
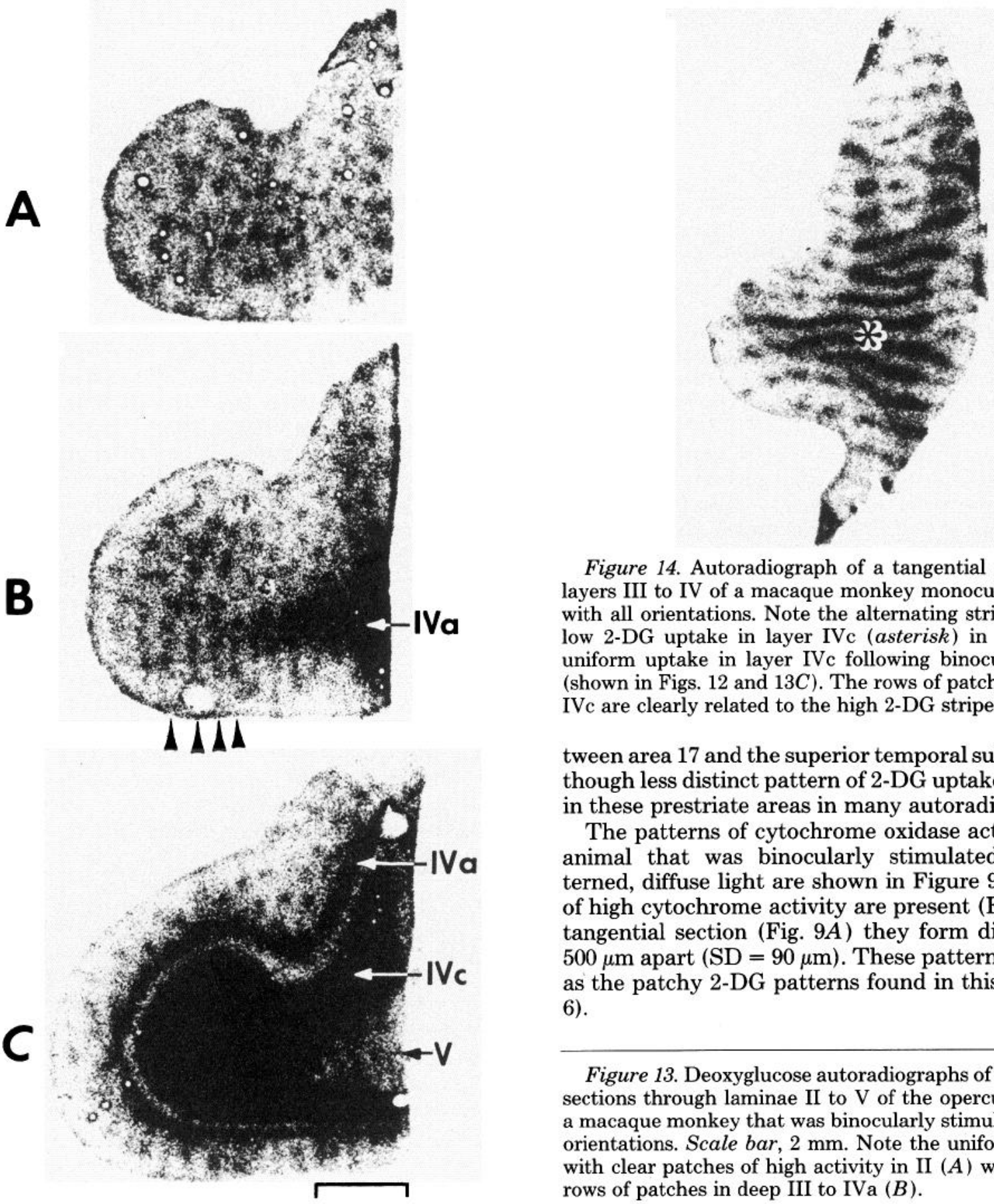

Figure 14. Autoradiograph of a tangential section through layers III to IV of a macaque monkey monocularly stimulated with all orientations. Note the alternating stripes of high and low 2-DG uptake in layer IVc (asterisk) in contrast to the uniform uptake in layer IVc following binocular stimulation (shown in Figs. 12 and $13 C$ ). The rows of patches outside layer IVc are clearly related to the high 2-DG stripes in IVc.

tween area 17 and the superior temporal sulcus. A similar, though less distinct pattern of 2-DG uptake was observed in these prestriate areas in many autoradiographs.

The patterns of cytochrome oxidase activity from the animal that was binocularly stimulated with unpatterned, diffuse light are shown in Figure 9. Radial zones of high cytochrome activity are present (Fig. $9 B$ ) and in tangential section (Fig. $9 A$ ) they form distinct patches $500 \mu \mathrm{m}$ apart $(\mathrm{SD}=90 \mu \mathrm{m})$. These patterns are the same as the patchy $2-\mathrm{DG}$ patterns found in this monkey (Fig. $6)$.

Figure 13. Deoxyglucose autoradiographs of three tangential sections through laminae II to $\mathrm{V}$ of the opercular cortex from a macaque monkey that was binocularly stimulated with many orientations. Scale bar, $2 \mathrm{~mm}$. Note the uniform label in IVc, with clear patches of high activity in II $(A)$ which merge into rows of patches in deep III to IVa $(B)$. 


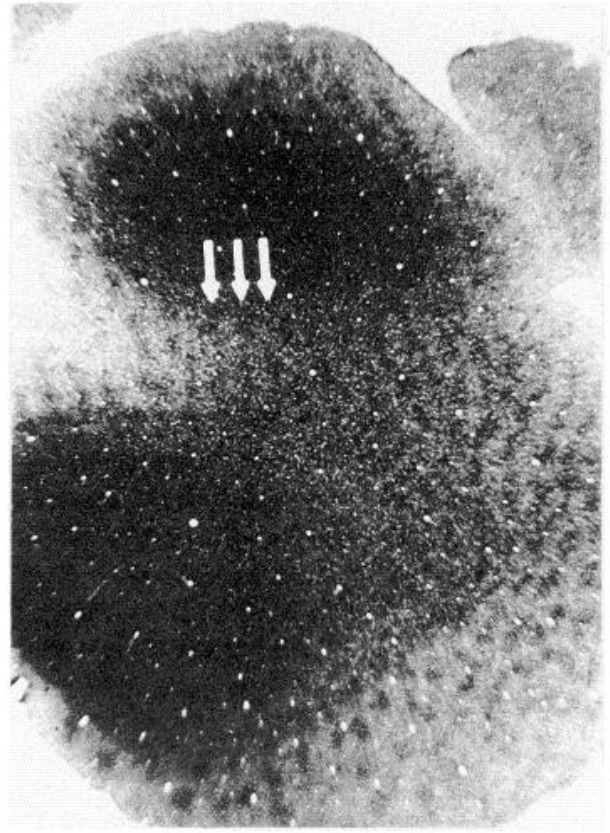

Figure 15. The patches-in-rows pattern of cytochrome oxidase reactivity in the superficial layers of normal macaque monkey area 17. The arrows mark three adjacent rows of patches spaced roughly $400 \mu \mathrm{m}$ apart.

Because the two metabolic labels produced similar patterns across visual cortex, we used double labeled sections to determine whether the labels mark the same cortical loci. Cryostat-cut sections were freeze-dried, apposed to $\mathrm{x}$-ray film to visualize the 2-DG pattern, and then reacted for cytochrome oxidase. Figure $10 \mathrm{~A}$ is a 2DG autoradiograph of a tangential section through the right striate cortex from the animal that was monocularly stimulated through the right eye while viewing diffuse light. The same section reacted for cytochrome oxidase appears in Figure $10 B$. The patches of high cytochrome oxidase activity in Figure $10 B$ are smaller and more distinct than the 2-DG patches in Figure $10 \mathrm{~A}$. Figure $10 \mathrm{C}$ is a composite drawing in which the solid circles indicate the locations where the cytochrome oxidase and 2-DG patches overlap. For $83 \%$ of the cytochrome oxidase patches there is a corresponding patch of high 2-DG uptake. A similar correspondence was found in the other hemisphere. In no case did we find a 2-DG patch unaccompanied by a cytochrome oxidase patch. The open circles in Figure $10 C$ indicate the locations where dark areas in the autoradiograph made it impossible to tell whether a distinct 2-DG patch was present. Similarly processed, double labeled sections from an animal that was binocularly stimulated with all orientations produced the same correspondence between the two labels. These findings demonstrate that the 2-DG and cytochrome oxidase labels mark the same layers and the same radial zones of striate cortex, although they do not allow us to identify whether the same neurons or types of neuronal structures are double labeled.

The $2 \mathrm{DG}$ and cytochrome oxidase labels appear to be dissociable under some stimulus conditions. Figure 11 shows a cytochrome oxidase-labeled section from the animal that was binocularly lid sutured 3 days before the
2-DG experiment. The 2-DG pattern in this animal's cortex was uniform (Fig. 7), but patches of high cytochrome oxidase activity, spaced $500 \mu \mathrm{m}$ apart, were still visible (Fig. 11). The continued presence of high cytochrome oxidase levels within these zones after 3 days of lid suture indicates that these zones still have significant capacity for enzymatic activity and that this activity is not rapidly responsive to visual deprivation.

\section{Macaca: Deoxyglucose and cytochrome oxidase patterns}

Radial zones of high 2-DG uptake have been demonstrated previously in the macaque striate cortex after patterned light stimulation. These zones have been attributed to ocular dominance columns (Kennedy et al., 1976; Hubel et al., 1978; Hendrickson and Wilson, 1979), orientation columns, or a combination of the two (Hubel et al., 1978). In none of these earlier studies in the macaque have controls similar to those just described been done to determine whether any base line patterns of oxidative metabolism exist which might confound the interpretation of 2-DG results in this species. We will include here the results from an earlier series of unpublished experiments for the sake of comparison with our Saimiri results.

Two juvenile Macaca nemestrina were administered 2-DG while they binocularly viewed a complex laboratory scene which contained all colors and orientations, as well as moving people. These stimulus conditions allowed a direct comparison with the earlier Macaca studies using binocular and monocular stimulation (Kennedy et al., 1976; Hendrickson and Wilson, 1979).

An autoradiograph from a section through the striate cortex of one of these macaque monkeys is shown in Figure 12. Layer IVc is continuously and heavily labeled as was expected from binocular stimulation. However, radial zones of high 2-DG uptake are still present above and below layer IVc. The zones are most distinct in layers II to III and IV. Layer IVa shows a distinct patchy irregularity superimposed on a high background of uptake. Figure 13 shows autoradiographs of tangential sections through layers II to V. When viewed from above, the radial zones form a dots-in-rows pattern. In layers II and III (Fig. 13A) there are distinct patches of high uptake, aligned into rows. Regions of high 2-DG uptake form bridges between the distinct patches along one axis. These bridges become more pronounced in the lower part of layer III (Fig. 13B), giving the appearance of bands of heightened uptake. In the direction parallel to the bands, individual patches are spaced about $500 \mu \mathrm{m}$ apart, while perpendicular to the bands, patch spacing is about 350 to $400 \mu \mathrm{m}$. The patches disappear in layer IVc, which is densely labeled throughout, and reappear in layer V (Fig. 13C) and layer VI (not shown). The bands of interconnected patches run perpendicular to the 1718 border. This tangential pattern of label differs from that in the squirrel monkey (Figs. 3 and 4), where binocular or monocular stimulation with many orientations yielded only separate patches of high 2-DG uptake with a spacing of $500 \mu \mathrm{m}$ regardless of the axis of measurement. Aside from this species' difference, however, it is clear that in Macaca, as in Saimiri, radial zones of high 2-DG 
uplake are sill present in area 17 after binocular stimulation with many orientations.

Unlike the squirrel monkey, monocular stimulation in the macaque produces a pattern of deoxyglucose uptake different from that seen after binocular stimulation. Figure 14 shows an autoradiograph of a tangential section through area 17 of a Macaca that was stimulated monocularly under identical conditions while viewing a laboratory scene (Hendrickson and Wilson, 1979). In layer IVc alternate rows of high and low 2-DG uptake form distinct parallel stripes, in contrast to the uniform labeling in this layer seen after binocular stimulation. In the layers above IVc, radial zones of alternating high and low 2 -DG label are in register with the layer IVc stripes; that is, only every other row of zones is labeled after monocular stimulation in Macaca, as would be expected if the 2-DG were labeling ocular dominance columns.

A cytochrome oxidase-reacted section from a normal juvenile Macaca nemestrina is shown in Figure 15. In this tangential view distinct patches can be seen with bridgelike connections, forming a patches-in-rows pattern identical in spacing and trajectory to the 2-DG pattern illustrated in Figure 13. Unlike the 2-DG pattern, the cytochrome oxidasc pattern is the same whether the animal has been stimulated binocularly or monocularly.

\section{Discussion}

Our main conclusion from this study is that there are radial zones of high metabolic activity that are fixed in position at regular intervals across squirrel monkey striate cortex. These zones are demonstrated independently with either deoxyglucose autoradiography or cytochrome oxidase histochemistry, and double label experiments show that the labeled zones are coincident. While these two labels mirror regional differences in the levels of oxidative metabolism within the brain, the labels reflect different aspects of that metabolism. The cytochrome oxidase stain detects ongoing enzymatic activity in mitochondria and hence reflects differences between cells in their capacity for oxidative metabolism (WongRiley, 1976, 1979). Changes in cytochrome oxidase activity levels within cells occur slowly ( 1 to 4 weeks or more) over time (Wong-Riley, 1979; Wong-Riley et al., 1978). The deoxyglucose label detects regional differences in brain glucose uptake which reflect cellular metabolic activity; this more dynamic marker reflects changes in metabolism over a shorter period of time (namely, the 45 min of visual stimulation) (Sokoloff et al., 1977).

In our squirrel monkeys the pattern and spacing of the radial zones of high cytochrome oxidase were constant across all conditions of visual stimulation, including the 3 days of binocular deprivation. This constancy indicates that these radial zones are probably a basic component of the functional architecture within normal monkey visual cortex. The deoxyglucose results from Saimiri show that in the absence of light stimulation, metabolism within the zones is not noticeably higher than in surrounding regions (Fig. 7). The zones increase their relative activity in response to activation of the visual system either by a patterned stimulus or by diffuse light viewed either monocularly or binocularly.

We have not done all of the control experiments in the macaque monkey that were done for the squirrel monkey, but our results and those of others indicate similar metabolically active zones across macaque area 17 under conditions that have been assumed to be "base line." For instance, we found a dots-in-rows pattern of 2-DG uptake, identical to the cytochrome oxidase pattern, after binocular stimulation with many orientations. This nonuniform uptake was unexpected because in a similar control experiment by Kennedy et al. (1976) such a pattern was not reported. As well, Tootell and Silvcrman (1981) recently reported that binocular stimulation of a macaque monkey in a ganzfeld produced a somewhat patchy or "mottled" uptake of 2-DG in layer III, although the patches were of lower contrast than those in our squirrel monkeys. As in the squirrel monkey, the 2-DG patches did appear to superimpose on the cytochrome oxidase patches. Therefore we view these radial zones in both species as a form of 2-DG "background" because they appear after many types of visual stimulation including unpatterned light. As will be argued below, this means it is necessary not only to take take them into account, but to subtract their contribution to the overall 2-DG activity in order to extract the real 2-DG pattern caused by a specific visual stimulus.

Comparison with previous studies. Both squirrel and macaque monkeys exhibit similar laminar variations in deoxyglucose uptake within area 17 except for layer IV. Binocular stimulation causes layers IVa and IVc in both species to display relatively continuous high level activity (Figs. 1 to 3,12 , and 13), while monocularly stimulated macaque shows marked alternating high and low uptake. Layer IVb appears as a region of lower label sandwiched between the two denser sublaminae. IJptake within the radial zones generally appears to be highest within layers III, IVa, and VI, and is somewhat lower in layers IVb and $\mathrm{V}$ (Figs. 2 and 12). These laminar variations in uptake differ somewhat from those reported in two previous deoxyglucose studies of the macaque striate corlex. Kennedy et al. (1976) used awake monkeys exposed to binocular stimulus conditions similar to those that we used for the macaque. They reported that in some animals all of layer IV (i.e., IVa, b, and c) was densely and continuously labeled, while in other animals layer IVa appeared as a thin dark band which, in their Figure $2 A$, appears to be separaled from the lower part of the layer IV complex by a sublamina of low uptake which could be layer IVb. This trilaminate appearance of label is similar to what we generally observe in our monkeys. We cannot explain their findings that all of layer IV in some of their monkeys was labeled. Hubel et al. (1978) used anesthetized, paralyzed macaque monkeys and observed only a single lamina of continuous high label, corresponding to layer IVc, after binocular stimulation. Further, the columns of heightened uptake above layer IVc in their animals were most dense in layer IVb. We cannot account for these interlaboratory differences in laminar uptake patterns except to question whether the metabolic activity of different laminae may be affected differentially by attention, anesthesia, or paralysis.

Relationship of the radial zones to ocular input. The pattern and spacing of the radial zones of high base line metabolism in squirrel and macaque monkey area 17 are 
different and this probably reflects a fundamental difference in the ocular dominance organization in the two species. The macaque has well segregated ocular dominance columns (Hubel and Wiesel, 1972, 1978; LeVay et al., 1975; Hubel et al., 1977; Hendrickson et al., 1978). The radial zones of high deoxyglucose and cytochrome oxidase activity found in the macaque after stimulation with all orientations (Figs. 12 to 15 ) were recently shown (Horton and Hubel, 1981; Hendrickson et al., 1981) to lie in register with the centers of the ocular dominance slabs in layer IVc. Further, the monocular stimulation results (Fig. 14) indicate that each row of dots is activated preferentially by one eye, since 2-DG uptake in one row is significantly higher than in the adjacent rows. This conclusion is in agreement with that of Horton and Hubel (1981)

The squirrel monkey, like most New World monkeys, does not appear to have ocular dominance columns. After monocular injections of $\left[{ }^{3} \mathrm{H}\right]$ proline into squirrel monkeys, layer IVc was completely labeled, indicating that the lateral geniculate nucleus afferents from each eye overlap substantially (Hubel et al., 1976; Tigges et al., 1977; Hendrickson et al., 1978). In addition, puffs of ${ }^{3} \mathrm{H}$ labeled protein, spaced $500 \mu \mathrm{m}$ apart, were seen in layer III after the eye injections. It could not be determined in those experiments whether the puffs were due to a direct geniculate input to layer III or were due to trans-synaptic transport from cells in layer IVc. Recent data from Hubel and Livingstone (1981) and Fitzpatrick ct al. (1983) favor a direct geniculate involvement because the puffs are clearly seen after injections of wheat germ agglutininconjugated horseradish peroxidase (WGA-HRP) or $\left[{ }^{3} \mathrm{H}\right]$ proline into the LGN. Interestingly, in squirrel monkeys, it appears to be cells in the interlaminar zones and/or $\mathrm{S}$ layers of the geniculate which give rise to the layer III puffs, since small injections of $\mathrm{HRP}$ that are restricted to layers I to III of area 17 only label cells in these geniculate layers (Fitzpatrick et al., 1983). The puffs in cortex do not appear to represent eye dominance patches because their spacing does not change after large injections of WGA-HRP that include all of the deep interlaminar zones and $\mathrm{S}$ layers of the geniculate (Fitzpatrick et al., 1983; D. Fitzpatrick, personal communication). Puffs of label are also seen in layer III of the macaque after injections of $\left[{ }^{3} \mathrm{H}\right]$ proline into the deep layers of the LGN, but the puffs are reported to be much less distinct than in Saimiri, and it is not clear where the cells giving rise to the puff's are located in the geniculate (Hubel and Livingstone, 1981).

During electrophysiological recording in squirrel monkey area 17, most cells in all of the laminae can be activated binocularly (Hubel and Wiesel, 1978). However, it has been reported that despite the strong binocularity, periodic fluctuations in eye dominance can be found roughly every $250 \mu \mathrm{m}$ on tangential electrode penetrations across Saimiri area 17 (Hubel and Wiesel, 1978). It is conceivable that these fluctuations reflect a modified or weak patchy organization for ocular dominance in this species, but their $250-\mu \mathrm{m}$ spacing is difficult to reconcile with the $500-\mu \mathrm{m}$ spacing of the radial zones of high metabolism shown here. This discrepancy in spacing suggests that the radial zones seen here in Saimiri do not represent left and right eye preference columns, as they do in Macaca. This conclusion is further supported by our finding that the spacing of the radial zones of high 2DG uptake in squirrel monkey does not change when one or both eyes are stimulated (cf. Figs. 4 and 5), in marked contrast to the change seen in the macaque under similar conditions (cf. Figs. 13 and 14). In the cat and macaque monkey, the cytochrome oxidase stain has been used to reveal ocular dominance columns that are associated with the remaining eye after enucleation or lid suture of the other eye (Wong-Riley, 1979; Horton and Hubel, 1981). We recently examined the cytochrome oxidase staining of striate cortex from a squirrel monkey that had been monocularly enucleated for $2^{+}$years and found no changes in the continuous staining of layer IV or in the pattern and spacing of the radial zones (A. E. Hendrickson and M. Tigges, unpublished observations). Taken together, all of these data indicate that cells in the radial zones of high metabolism as well as in layer IV of squirrel monkey striate cortex are driven roughly equally by both eyes. These results also provide further evidence that the squirrel monkey does not have ocular dominance columns, at least like those in the macaque monkey.

Problems the background zones cause for interpreting 2-DG patterns. In both squirrel and macaque monkey, patchy patterns of high deoxyglucose uptake are seen after binocular stimulation with all orientations. These patterns change to a band- or lattice-like network of high 2-DG uptake after binocular stimulation with a single orientation (Fig. 3; Hubel et al., 1978; Horton and Hubel, 1981; Tootell and Silverman, 1981). Horton and Hubel (1981) have reported for the macaque monkey that the lattice of high 2-DG which resulted from vertical or horizontal stripe stimulation invariably encompassed the cytochrome oxidase patches. Also, "hot spots" of very high 2-DG uptake generally overlapped with the cytochrome patches. A similar correspondence between the $2-D G$ and cytochrome labels may occur in squirrel monkeys stimulated with one orientation, although additional animals are necessary to confirm this. Because the cytochrome patches correspond to the background zones of high metabolism, their coincidence with the 2-DG hot spots in the lattice suggests that the lattice pattern may reflect two superimposed patterns, one due to the constant stripe orientation and the other due to patches of high metabolism. If this is the case, then one cannot easily infer from the autoradiographs alone how orientation-selective cells are distributed across monkey visual cortex. Hubel and Livingstone (1981) have found by electrophysiological recording that the patches of high cytochrome oxidase activity contain clusters of nonorientation-selective cells, many of which respond to diffuse light, while oriented cells occupy the surrounding regions. Their finding suggests that, after a monkey views a single orientation, the 2-DG-labeled hot spots contain nonoriented cells, while the remainder of the band/lattice contains cells selective for the stimulus oricntation (Hubel and Livingstone, 1981). However, it is not yet clear whether the hot spots exclusively contain only nonoriented cells or whether the remaining regions of the lattice mark the locations of all of the cells tuned to the stimulus orientation. Combined microelectrode and 2-DG studies 
in the monkey, such as are being done in the cat (Schoppmann and Stryker, 1981), are needed to confirm the latter point.

The finding that the cytochrome patches contain nonoriented cells (Hubel and Livingstone, 1981) explains why patches of high 2-DG uptake persist in monkey visual cortex after stimulation with diffuse light or with bars of all orientations in that these cells are probably activated by all of the bar orientations and/or by diffuse light. Further, Tootell and Silverman (1981) have reported that a complex lattice-like pattern of high 2-DG uptake was produced after a monkey binocularly viewed a bar pattern having many orientations but only high spatial frequencies. The cytochrome oxidase patches overlapped the regions of low 2-DG uptake between the lattice rather than being coincident with the lattice. These results suggest that the LGN afferents and/or the cells within the cytochrome oxidase patches may respond mainly to low spatial frequencies and poorly or not at all to high frequencies (Tootell and Silverman, 1981). Taken together, these physiological and deoxyglucose data indicate that an oriented stimulus, which contains both low and high spatial frequencies, might be expected to activate nonoriented cells within the cytochrome patches as well as orientation-selective cells outside the patches. The resulting lattice of 2-DG uptake would reflect both the regions of oriented and nonoriented cells and thus would not accurately reflect the distribution of cells tuned to the stimulus orientation. The pattern of stripes used in this study and that of Hubel and co-workers contained both low and high spatial frequencies and thus the resulting 2-DG patterns of heightened uptake probably reflect both "nonspecific" and orientation-specific uptake.

In a related problem, Hubel et al. (1978) obtained a dotlike 2-DG pattern in the superficial layers of a macaque monkey after the animal was monocularly stimulated with vertical stripes (Figs. $10 \mathrm{~A}$ and 11 of Hubel et al., 1978). They interpreted this as revealing the distribution of cells tuned to vertical and driven by the open eye. However, their patchy pattern appears to be indistinguishable from the dots-in-rows pattern shown here (Fig. 13) after monocular stimulation with all orientations (also see Kennedy et al., 1976). It is possible that the patches shown by Hubel et al. (1978) simply represent the radial zones of high metabolism that are associated with nonspecific monocular stimulation.

The background patches of increased 2-DG uptake and cytochrome oxidase that are present in the monkey are not present in area 17 of the tree shrew (Humphrey et al., 1980; Horton and Hubel, 1981) or cat (Schoppmann and Stryker, 1981; Wong-Riley, 1979), and thus stimulusinduced patterns of 2-DG uptake are more readily interpretable in these species. The absence of zones of high background metabolism in the tree shrew and cat may reflect fundamental differences between these species and the monkey in either the topographic distribution of oriented and nonoriented cells within area 17 or differences in the functional organization for the processing of other stimulus parameters.

Relationship of the hackground zones to cortical inhibition. Hendrickson (1982; Hendrickson et al., 1981) has recently shown that the localization of the GABAsynthesizing enzyme glutamic acid decarboxylase (GAD) is identical to the distribution of cytochrome oxidase activity in macaque area 17. Layers II and III contain distinct zones of high GAD activity which superimpose on the cytochrome oxidase patches. Both GAD-positive somata and axon terminals are more heavily concentrated in these zones than in surrounding regions. Ongoing experiments have shown that a similar laminar and radial pattern of GAD and cytochrome activity also exists in squirrel monkey area 17 (not illustrated). At present, it is not clear how to interpret these findings concerning GAD. Because the radial zones as well as layer IV contain large numbers of GABAergic terminals, these terminals may significantly increase the local metabolic levels (Wong-Riley, 1976). On the other hand, some of the increased 2-DG/cytochrome/GAD activity in the radial zones may be due to the direct geniculate input to layer III, resulting in increased synaptic density in the radial zones. In addition to this proposed difference in structural organization across layer III, the nonoriented cells in the radial zones would be expected to discharge irrespective of stimulus orientation, making them more tonically active than the orientation-selective cells surrounding them, resulting in a higher overall metabolism needing higher enzyme levels. All of these various possibilities require additional study to truly explain this periodic organization.

Functional implications. The overall significance of the radial zones for cortical function is still not clear. In the macaque, cells in the zones appear to be preferentially related to one eye. The zones are in register with the ocular dominance columns in layer IV, they are strongly monocular, and their metabolic levels are modulated by stimulation through the proper eye. Such stimulation might generate strong within-column inhibition that could suppress input to cells in the column from the nondominant cyc. As suggested by Sillito et al. (1980), this inhibition could serve as a neural mechanism for stereopsis. However, the radial zones are present in all regions of the visual cortex including those representing very peripheral areas of the visual field which are monocular, and this suggests that their role in precise binocular vision may be limited. In Saimiri the radial zones are well activated by either eye, which seems to rule out their involvement in nondominant eye inhibition. Whether these zones are closely related to the orientation column system or to other features of visual cortical architecture remains to be determined.

Note added in proof. Hubel and Livingstone (1982) have recently reported that a large proportion of neurons in the layer III patches of high cytochrome oxidase activity have receptive fields that are organized for color opponency and that these cells appear to be restricted to the patches.

\section{References}

Albus, K. (1979) $\left[{ }^{14} \mathrm{C}\right]$ Deoxyglucose mapping of orientation subunits in the cat's visual cortical areas. Exp. Brain Res. 37: 609-613. 
Cowey, A. (1964) Projection of the retina onto striate and prestriate cortex in squirrel monkey, Saimiri scireus. J. Neurophysiol. 27: 366-393.

Fitzpatrick, D., K. Itoh, and I. T. Diamond (1983) The laminar organization of the lateral geniculate body and the striate cortex in the squirrel monkey (Saimiri sciureus). J. Neurosci., in press.

Flood, D. G., and P. D. Coleman (1979) Demonstration of orientation columns with $\left[{ }^{14} \mathrm{C}\right]-2$-deoxyglucose in a cat reared in a striped environment. Brain Res. 173: 538-542.

Hendrickson, A. E. (1982) The orthograde axoplasmic transport tracing technique and its implications for additional striate cortex neuroanatomical analysis. In Cytochemical Methods in Neuroanatomy, V. Chan-Palay and S. Palay, eds., Alan R. Liss, Inc., New York.

Hendrickson, A. E., and J. R. Wilson (1979) A difference in $\left[{ }^{14} \mathrm{C}\right]$ deoxyglucose autoradiographic patterns in striate cortex between Macaca and Saimiri monkeys following monocular stimulation. Brain Res. 170: 353-358.

Hendrickson, A. E., J. R. Wilson, and M. P. Ogren (1978) The neuroanatomical organization of pathways between the dorsal lateral geniculate nucleus and visual cortex in Old World and New World primates. J. Comp. Neurol. 182: 123-136.

Hendrickson, A. E., S. P. Hunt and J. -Y. Wu (1981) Immunocytochemical localization of glutamic acid decarboxylase in monkey striate cortex. Nature 292: 605-607.

Horton, J., and D. H. Hubel (1981) Regular patchy distribution of cytochrome oxidase staining in primary visual cortex of macaque monkey. Nature 292: 762-764.

Hubel, D. H., and M. S. Livingstone (1981) Regions of poor orientation tuning coincide with patches of cytochrome oxidase staining in monkey striate cortex. Soc. Neurosci. Abstr. 7: 367 .

Hubel, D. H., and M. S. Livingstone (1982) Cytochrome oxidase blobs in monkey area 17: Response properties and afferent connections. Soc. Neurosci. Abstr. 8: 706.

Hubel, D. H., and T. N. Wiesel (1968) Receptive fields and functional architecture of monkey striate cortex. J. Physiol. (Lond.) 195: 215-243.

Hubel, D. H., and T. N. Wiesel (1972) Laminar and columnar distribution of geniculo-cortical fibers in the macaque monkey. J. Comp. Neurol. 146: 421-450.

Hubel, D. H., and T. N. Wiesel (1978) Distribution of inputs from the two eyes to striate cortex of squirrel monkeys. Soc Neurosci. Abstr. 4: 632.

Hubel, D. H., T. N. Wiesel, and S. LeVay (1976) Functional architecture of area 17 in normal and monocularly deprived macaque monkey. Cold Spring Harbor Symp. Quant. Biol. 40: $581-589$.

Hubel, D. H., T. N. Wiesel, and S. LeVay (1977) Plasticity of ocular dominance columns in monkey striate cortex. Philos. Trans. R. Soc. Lond. (Biol.) 278: 377-409.

Hubel, D. H., T. N. Wiesel, and M. P. Stryker (1978) Anatomical demonstration of orientation columns in macaque monkey. J. Comp. Neurol. 177: 361-379.
Humphrey, A. L., and A. E. Hendrickson (1980) Radial zones of high metabolic activity in squirrel monkey striate cortex. Soc. Neurosci. Abstr. 6: 315.

Humphrey, A. L., and T. T. Norton (1980) Topographic organization of the orientation column system in the striate cortex of the tree shrew (Tupaia glis). I. Microelectrode recording. J. Comp. Neurol. 192: 531-547.

Humphrey, A. L., L. C. Skeen, and T. T. Norton (1980) Topographic organization of the orientation column system in the striate cortex of the tree shrew (Tupaia glis). II. Deoxyglucose mapping. J. Comp. Neurol. 192: 549-566.

Kennedy, C., M. H. Des Rosiers, O. Sakurada, M. Shinohara, M. Reivich, H. W. Jehle, and L. Sokoloff (1976) Metabolic mapping of the primary visual system of the monkey by means of the autoradiographic $\left[{ }^{14} \mathrm{C}\right]$ deoxyglucose technique. Proc. Natl. Acad. Sci. U. S. A. 73: 4230-4234.

LeVay, S., D. H. Hubel, and T. N. Wiesel (1975) The pattern of ocular dominance columns in macaque visual cortex revealed by a reduced silver stain. J. Comp. Neurol. 159: 559-576.

Lund, J. S. (1973) Organization of neurons in the visual cortex, area 17, of the monkey (Macaca mulatta). J. Comp. Neurol. 147: 455-496.

Schoppmann, A., and M. P. Stryker (1981) Physiological evidence that the 2-deoxyglucose method reveals orientation columns in cat visual cortex. Nature 293: 574-576.

Sillito, A. M., J. A. Kemp, and H. Patel (1980) Inhibitory interactions contributing to the ocular dominance of monocularly dominated cells in the normal cat striate cortex. Exp. Brain Res. 41: 1-10.

Sokoloff, L., M. Reivich, C. Kennedy, M. H. Des Rosiers, C. S. Patlak, K. D. Pettigrew, O. Sakurada, and M. Shinohara (1977) The $\left[{ }^{14} \mathrm{C}\right]$ deoxyglucose method for the measurement of local cerebral glucose utilization: Theory, procedure, and normal values in the conscious and anesthetized albino rat. J. Neurochem. 28: 897-916.

Stryker, M. P., D. H. Hubel, and T. N. Wiesel (1977) Orientation columns in the cat's visual cortex. Soc. Neurosci. Abstr. 3: 1852.

Tigges, J., M. Tigges, and A. A. Perachio (1977) Complementary laminar terminations of afferents to area 17 originating in area 18 and in the lateral geniculate nucleus in squirrel monkey. J. Comp. Neurol. 176: 87-100.

Tootell, R. B. H., and M. S. Silverman (1981) A comparison of cytochrome oxidase and deoxyglucose patterns in macaque visual cortex. Soc. Neurosci. Abstr. 7: 366.

Wong-Riley, M. (1979) Changes in the visual system of monocularly sulured or enucleated cats demonstrable with cytochrome oxidase histochemistry. Brain Res. 171: 11-28.

Wong-Riley, M. T. T. (1976) Endogenous peroxidatic activity in brain stem neurons as demonstrated by their staining with diaminobenzidine in normal squirrel monkeys. Brain Res. 108: $257-277$.

Wong-Riley, M. T. T., M. M. Merzenich, and P. A. Leake (1978) Changes in endogenous enzymatic reactivity to DAB induced by neuronal inactivity. Brain Res. 141: 185-192. 